\title{
L'appariement forme-fonction en FL2 : variation des combinaisons de conjugaisons dans la narration au passé
}

\author{
Marie-Eve Michot $^{1}$, et Michel Pierrard ${ }^{1}$ \\ ${ }^{1}$ Vrije Universteit Brussel - CLIN Multi-L, Belgique
}

Résumé. La mise en relation d'une forme et d'une fonction représente une dimension clef du processus d'acquisition d'une langue seconde (L2). L'appariement de celles-ci se met en place progressivement car elles ne sont pas indissociablement liées dès le début du processus développemental (Perdue \& Klein 1992 ; Perdue et al. 2002). Sur un plan général, le processus acquisitionnel passe d'un stade premier de lexicalisation vers un stade de morphologisation, qui ouvre la perspective d'un emploi adéquat, d'un appariement donc entre la forme et sa/ ses fonction(s), et vice versa (Noyau 1997). Le développement tardif de l'appariement forme-fonction d'une L2 a bien été démontré par diverses études ayant analysé l'emploi du passé composé et de l'imparfait par des apprenants FL2 (cf. e.a. Kaplan 1987; Noyau et al. 1995 ; Kihlstedt 2002 ; Ayoun 2001, 2004). Peu d'études ont néanmoins examiné l'appariement forme-fonction du passé chez des apprenants avancés ou quasi-natifs. Cet article a pour objectif d'examiner l'emploi progressif des conjugaisons du passé en FL2 et d'identifier les particularités de leur emploi fonctionnel à un niveau avancé en comparant les productions d'apprenants avec celles de natifs.

\begin{abstract}
Form-function mapping in FL2: knowledge and usage of the conjugations of the past. The appropriate form-function mapping represents a key dimension in the L2 acquisition process. The mapping of form and function are not inextricably linked from the beginning of the developmental process onwards (Perdue \& Klein 1992; Perdue et al. 2002). In sum, the acquisitional process evolves from a first lexicalisation stage to a morphological stage, which enables the appropriate form-function mapping (Noyau 1997). Several studies have already confirmed the late development of form-function mapping regarding the use of passé composé and imparfait by L2 learners (cf. i.a. Kaplan 1987; Noyau et al. 1995 ; Kihlstedt 2002; Ayoun 2001, 2004). Nevertheless, few studies have examined the form-function mapping of advanced or native-like L2 learners. This article reports on the progressive use of the verbal forms of the past in FL2 and on the characteristics of the functions expressed by these forms at an advanced level, by comparing the productions of L2 learners and native speakers.
\end{abstract}




\section{Introduction}

Comme Klein (1995) l'a indiqué, une dimension clef du processus d'acquisition d'une langue seconde (L2) est la réorganisation continuelle des différents moyens d'expression dont dispose l'apprenant. Celui-ci cherche la/ les forme(s) L2 adéquate(s) pour exprimer une certaine fonction qui lui est déjà connue grâce à son expérience langagière antérieure (Véronique 2009: 302). L'appariement d'une forme et d'une fonction se met en place progressivement car celles-ci ne sont pas indissociablement liées dès le début du processus développemental (Perdue \& Klein 1992 ; Perdue et al. 2002). Ainsi, l'absence de marques morphologiques dans l'usage n'indique pas automatiquement une absence d'indications fonctionnelles dans l'interlangue de l'apprenant (p. ex. l'emploi d'adverbes temporels peut compenser l'absence de morphologie verbale pour marquer des contrastes temporels). De même, la présence d'une forme morphologique n'implique pas nécessairement la maitrise de sa fonction ou de l'une des fonctions qui lui est attribuée dans la langue cible (p. ex. son emploi précoce en tant que «chunk» ou structure formulaïque). Dans le processus acquisitionnel, l'emploi d'une forme témoigne d'importants développements concernant d'une part la précision de son appariement avec une certaine fonction (un certain sens), et de l'autre de l'extension des fonctions qu'elle exprime. Ces développements sont liés (a) à l'extension du répertoire de formes accessibles dans l'interlangue de l'apprenant, qui modifie l'équilibre entre les formes et leurs fonctions et (b) à la progression de la grammaticalisation qui affine l'appariement forme-fonction dans le discours de l'apprenant (cf. le « one-to-one principle » et le «multifunctionality principle » (Andersen 1984, 1990)).

C'est dans ce cadre que la présente étude examine l'emploi des formes verbales pour exprimer le passé. L'appariement forme-fonction adéquat s'y développe tardivement, comme cela a été démontré par diverses études ayant analysé l'emploi du passé composé et de l'imparfait par des apprenants FL2 (cf. e.a. Kaplan 1987 ; Noyau et al. 1995 ; Kihlstedt 2002 ; Ayoun 2001, 2004). Cet article, qui compare le développement des formes verbales du passé chez des apprenants FL2 avec l'emploi de ces formes par des natifs dans la réalisation de la même tâche narrative, a pour objectif

(a) de décrire jusqu'à quel point l'ajustement du rapport forme-fonction va s'opérer dans les productions des apprenants pour les différentes conjugaisons du passé (« form-tofunction »)

(b) et d'évaluer dans quelle mesure la disponibilité d'autres conjugaisons pour les mêmes types de fonctions va diversifier les formes utilisées et les combinaisons possibles de ces conjugaisons dans divers types de scénarios pour narrer la même histoire (« function-to-form »).

\section{Cadre théorique}

Diverses études ont étudié la maîtrise progressive des conjugaisons du passé du point de vue de la relation «form-to-function », en particulier celle du passé composé et de l'imparfait (e.a. Kihlstedt 2002; Ayoun 2004 ; McManus 2015). L'étude de l'appropriation des conjugaisons du passé s'effectue plus spécifiquement sur l'arrière-fond de deux théories qui s'opposent quant à la primauté du marquage fonctionnel par la morphologie verbale du passé : l'hypothèse aspectuelle (Aspect Hypothesis) et l'hypothèse du temps passé par défaut (Default Past Tense Hypothesis) (cf. e.a. Labeau 2005 ; Michot \& Pierrard 2016).

Selon l'hypothèse aspectuelle d'Andersen $(1986,1991)$, l'apprenant L2 utilise dans un premier temps la flexion verbale pour exprimer une perspective aspectuelle avant de l'exploiter pour indiquer le temps. Elle est concrétisée sous la forme de 4 hypothèses (Li \& Shirai $2000: 50)$ : 
1. Les apprenants marquent d'abord le perfectif ${ }^{1}$ (le "passé composé » pour le français) sur des verbes d'achèvement (ex. apercevoir) et d'accomplissement (ex. traverser), qui sont téliques car leur procès inhérent a un point final.

2. L'imperfectif (« imparfait » en français) apparaît après le perfectif.

3. Les premières distinctions imperfectives sont marquées sur des verbes d'état (ex. savoir) et des verbes d'activité (ex. courir), qui eux sont atéliques, leur procès inhérent n'ayant pas un point final.

4. Le marquage du progressif (par exemple formes en -ing en anglais) se fait d'abord sur les verbes d'activité, s'étend ensuite aux verbes d'accomplissement et d'achèvement et rarement aux verbes d'état.

L'hypothèse du temps passé par défaut de Salaberry $(1999,2003)$ postule, contrairement à celle d'Andersen, que les distinctions temporelles précèdent celles de l'aspect, étant donné que les apprenants utilisent initialement un seul temps du passé, à savoir le passé composé : « [learners] will initially rely on a single marker of past tense, most typically the perfective form » (Salaberry \& Ayoun 2005: 20). L'impact de l'aspect est plus tardif selon cette hypothèse car seuls les apprenants avancés ont recours à l'aspect pour discriminer l'usage du passé composé et de l'imparfait.

Une troisième hypothèse prétend cependant expliquer l'emploi des conjugaisons du passé, sans s'engager sur l'antériorité du temps ou de l'aspect : l'hypothèse discursive (Discourse Hypothesis) de Bardovi-Harlig (1994), qui indique que les apprenants utilisent la morphologie verbale pour distinguer le premier plan de l'arrière-plan dans leurs narrations. Selon cette hypothèse, le perfectif est ainsi utilisé pour encoder le premier plan et l'imperfectif pour l'arrière-plan.

Pour le français L2, les études d'Ayoun $(2001,2004)$ en particulier ont examiné 1'acquisition du système temporo-aspectuel FL2 en se concentrant sur l'utilisation du passé composé et de l'imparfait. Son corpus est composé de données expérimentales et de productions écrites élicitées auprès d'apprenants anglophones universitaires. L'étude de 2001 constate un développement dans l'usage du passé composé, mais pas dans celui de l'imparfait. Ayoun explique ce résultat par la plus grande complexité aspectuelle de l'imparfait, qui est utilisé pour marquer l'aspect imperfectif, mais aussi itératif et duratif (cf. aussi Kaplan 1987). Les apprenants de cette étude limitent leur emploi de l'imparfait à l'aspect duratif. Les productions contiennent donc déjà un appariement forme-fonction discriminant, mais l'emploi fonctionnel adéquat de l'imparfait est limité. L'étude de 2004 a pour but d'évaluer les hypothèses 2 et 3 de l'hypothèse aspectuelle (cf. supra). Ayoun conclut que les données confirment partiellement l'hypothèse d'Andersen (1986, 1991) étant donné que le développement du perfectif est antérieur à celui de l'imperfectif et que les apprenants associent effectivement les marques morphologiques avec différentes classes sémantiques, mais cependant pas selon la progression postulée par Andersen (Ayoun $2004: 43$ ).

Michot \& Pierrard (2016) ont observé le développement des conjugaisons du passé dans l'interlangue d'apprenants néerlandophones du FL2 afin d'examiner s'il y a primauté du marquage de l'aspect ou du temps. Sur la base des données recueillies, cette première étude confirme prudemment l'importance et la primauté du marquage de l'aspect dans l'interlangue des apprenants FL2, liée à la primauté des formes du passé composé par rapport à celles de l'imparfait dans la production des apprenants. Le passé composé y marque alors l'aspect accompli, face au présent qui indique l'inaccompli. Le surgissement de l'imparfait, qui est

\footnotetext{
${ }^{1}$ Nous définissons les termes perfectif et imperfectif de la manière suivante : « The perfective aspect is a grammatical aspect used to describe an action viewed as a simple whole- a unit without interior composition. The perfective aspect is distinguished from the imperfective aspect, which presents an event as having internal structure (such as ongoing, continuous, or habitual actions) » (Comrie 1976 : 12).
} 
nettement plus tardif, semble aller de pair avec la prise en compte de l'expression du temps passé par le passé composé et donc aussi de la nécessité d'introduire une dimension d'inaccompli au passé. L'importance de l'aspect lexical inhérent des verbes (cf. télicité/ atélicité) dans le surgissement des premières occurrences conjuguées au passé composé et à l'imparfait semble aussi modérément confirmée.

Du point de vue de la relation « function-to-form », les études concernant le français sont plus rares. Signalons toutefois les études de Kaplan (1987) et Noyau et al. (1995). Kaplan (1987) postule que l'apprenant possède au niveau débutant déjà un système aspectuel mais que celui-ci ne correspond pas au système français. Il remarque ainsi que la grande majorité des apprenants utilisent les formes du présent dans des contextes demandant l'imparfait.

Les résultats de l'étude de Noyau et al. (1995) ont montré que les distinctions morphologiques systématiques sont soit difficiles à acquérir, soit limitées aux stades les plus avancés du développement. La morphologie verbale utilisée par leurs apprenants permet des distinctions modales (V-ø vs V-e pour l'opposition mode realis vs irrealis) ou temporelles ( $\mathrm{V}-\varnothing$ et Aux-V-e pour distinguer le présent et le passé), tandis que la morphologie verbale utilisée par d'autres apprenants combine des marques temporelles et aspectuelles (V- $\varnothing$ vs e-V pour situations en cours, progressives vs situations finies au passé). La flexion verbale est présente, mais elle ne témoigne pas nécessairement d'un appariement forme-fonction conscient.

La présente recherche se centre sur deux aspects spécifiques de l'appariement formefonction dans le développement de la maîtrise des conjugaisons du passé : d'une part elle compare la production d'apprenants FL2 avec celle de natifs afin d'évaluer le degré d'ajustement du rapport forme-fonction dans les productions des deux groupes. D'autre part, elle examine comment les apprenants et les natifs combinent les différentes conjugaisons convoquées pour effectuer la narration demandée afin de déterminer dans quelle mesure la disponibilité d'autres conjugaisons pour les mêmes types de fonctions diversifie les formes utilisées et multiplie les combinaisons possibles des conjugaisons dans divers types de scénarios.

\section{Méthodologie et questions de recherche}

\subsection{Corpus}

Notre étude est fondée sur les données d'un corpus oral composé de trois groupes d'apprenants néerlandophones suivant des cours de FL2 dans l'enseignement secondaire flamand (partie néerlandophone de la Belgique) et de deux groupes de contrôle de locuteurs natifs. Les participants ont raconté l'histoire de la grenouille (Mayer 1969), une tâche narrative orale semi-spontanée couramment utilisée dans les études acquisitionnelles.

Les groupes d'apprenants se situent à des niveaux scolaires différents et présentent un nombre d'heures d'enseignement de FL2 croissant (cf. tableau 1). Le groupe NN1 est composé de 30 élèves de première année de secondaire (âgés de \pm 12 ans) ayant suivi environ 250 heures de FL2, le groupe NN3 représente 25 élèves ( \pm 14 ans) de troisième année ayant suivi environ 520 heures de FL2 et le troisième groupe, NN6, est composé de 32 élèves $( \pm 17$ ans) de la dernière année du secondaire qui ont suivi environ 710 heures de FL2. Les deux groupes de lycéens natifs FF1 et FF6 diffèrent par l'âge (12-13/ 17-18 ans) et par le niveau d'enseignement (première/ dernière année de l'enseignement secondaire). L'avantage du corpus utilisé est qu'il permet d'évaluer l'emploi des conjugaisons du passé (a) dans la production d'apprenants ayant acquis le FL2 dans un contexte éducatif et linguistique identique, c'est-à-dire avec des approches pédagogiques, un programme éducatif et des 
objectifs finaux imposés par le ministère de l'enseignement et (b) chez des locuteurs L1 qui proviennent également d'un contexte scolaire similaire aux apprenants mais où le français est bien sûr utilisé et enseigné comme L1. Par ailleurs, apprenants comme natifs effectuent la même tâche narrative.

Tableau 1. Présentation du corpus

\begin{tabular}{|l|c|c|c|c|c|c|}
\cline { 2 - 7 } \multicolumn{1}{c|}{} & \# d'apprenants & âge & $\begin{array}{c}\text { \# d'heures de } \\
\text { FL2 }\end{array}$ & $\begin{array}{c}\text { \# mots / } \\
\text { partic. }\end{array}$ & \# V / partic. & $\begin{array}{c}\text { \# V VAM/ } \\
\text { partic. }\end{array}$ \\
\hline NN1 & 30 & $+/-12$ & \pm 250 & 175,2 & 17,6 & 1,4 \\
\hline NN3 & 25 & $+/-14$ & \pm 520 & 245 & 27 & 4,8 \\
\hline NN6 & 32 & $+/-17$ & \pm 710 & 328,9 & 39,6 & 8,1 \\
\hline FF1 & 15 & $+/-12$ & natifs & 363,9 & 60,1 & 17,7 \\
\hline FF6 & 15 & $+/-17$ & natifs & 369,2 & 60,7 & 12,6 \\
\hline
\end{tabular}

\# d'apprenants :

nombre d'apprenants par groupe

\# d'heures de FL2 : nombre d'heures de cours de français langue seconde suivis en contexte scolaire

$\#$ mots/ partic. :

\# V/partic. :

\# $\mathrm{V}^{\mathrm{TAM}} /$ partic. : nombre moyen de mots produits par participant nombre moyen de verbes produits par participant nombre moyen de verbes marquant une nuance TAM produits par participant

En plus du nombre de participants par groupe et du nombre d'heures de FL2 suivies, le tableau 1 contient la production moyenne de mots, de verbes et de verbes marquant une nuance temporelle, aspectuelle ou modale (TAM) par participant. Les auxiliaires marquant des nuances TAM et toute forme verbale qui n'est pas conjuguée au présent ont été identifiés comme une marque TAM (par exemple devoir + inf., pouvoir + inf., être en train de + inf., cherchait, est tombé, etc.). Ces données indiquent un développement global progressif des productions des apprenants néerlandophones (NN), tant au niveau du nombre de mots, de verbes et de marques TAM produits (accroissement de NN1 à NN6 : mots x 1,90; verbes x 2,25 ; formes TAM x 5,80).

Le nombre moyen de mots et de verbes produits est comparable chez les deux groupes de natifs, mais ce n'est pas le cas pour le nombre moyen de marques TAM produites car les natifs plus âgés en produisent considérablement moins (12,6 marques par participant en moyenne dans FF6 par rapport à 17,7 dans FF1). Les données indiquent également que les productions des apprenants NN6 se rapprochent de celles des natifs pour le nombre moyen de mots produits, mais pas pour le nombre moyen de verbes ni pour le nombre moyen de marques TAM, qui sont toujours nettement plus élevés chez les natifs.

\subsection{Traitement des données}

Les productions orales ont été enregistrées et ensuite transcrites selon les conventions CHAT. Les données recueillies ont été traitées à l'aide du logiciel CLAN (The CHILDES Project, MacWhinney 2000) ${ }^{2}$ afin d'effectuer des calculs de fréquence.

Seules les formes verbales pouvant être indubitablement identifiées comme des marques TAM du français ont été incluses aux résultats présentés sous les points quatre et cinq. Quant à l'éventuelle prononciation problématique de l'imparfait des verbes en -ER (cf. Granget 2005, Thomas 2009), seules les claires prononciations en [ $\varepsilon$ ] (cf. ex. 1a) ont été identifiées comme des occurrences de l'imparfait. Les formes pour lesquelles la prononciation est douteuse ont été identifiées comme des formes non finies, dont le suffixe est transcrit par - $E$ (cf. ex. 1b).

(1) a. NN6-Sn13 : le soleil commençait à briller

b. NN6-823 : cette nuit la grenouille quittE sa bocal

${ }^{2}$ La majorité des codes CHAT n'ont pas été repris dans les exemples afin d'en faciliter la lecture. 
Les formes verbales que le participant reprend mot à mot de l'intervieweur ont été exclues des résultats (cf. ex. 2a). Quand un participant répète (indiqué par le symbole [/], cf. ex. 2b) ou reformule (indiqué par le symbole [//], cf. ex. 2c-d) une forme verbale, seule la dernière forme produite a été comptabilisée, que celle-ci soit correcte (cf. ex. 2c) ou incorrecte (cf. ex. $2 \mathrm{~d})$.

(2) a. NN1-227: *PUP: le petit garçon et le chien ils roepen@nld [= crier]

*INT: ils crient

*PUP: ils crient le grenouille

*PUP: ils cherchE [*]

b. NN3-805: *PUP: le chien pousse [/] pousse à l'arbre

c. NN3-805: *PUP: l'animal portE [//] porte le garçon

d. NN1-188: le cerf arrête [//] arrêtE [*]

Les occurrences extraites des transcriptions annotées ont été soumises à des analyses statistiques. Le test Kolmogorov-Smirnov a premièrement indiqué que les données ne sont pas normalement distribuées. Les données ont pour cette raison été soumises au test nonparamétrique Kruskal-Wallis afin de découvrir si les résultats des différents groupes varient de manière significative. Toutes les analyses statistiques ont été effectuées à l'aide du logiciel SPSS (24, IBM 2016).

\subsection{Questions de recherche}

La présente contribution se donne pour but de répondre aux questions de recherche suivantes

(1) sur le plan de l'ajustement du rapport forme-fonction :

Q1.1. quelle est l'évolution de la fréquence d'emploi des conjugaisons du passé dans la production des apprenants néerlandophones du FL2?

Q1.2. dans quelle mesure la fréquence d'emploi des conjugaisons du passé par les apprenants FL2 du groupe le plus avancé correspond-elle à celle des locuteurs natifs?

(2) sur le plan de la diversification du rapport fonction-forme :

Q2.1. quels sont les types de configuration temporelle exploités par les FL2 pour exprimer une narration au passé ?

Q2.2. dans quelle mesure les types de combinaisons de conjugaisons exploités par les apprenants FL2 du groupe le plus avancé pour exprimer une narration au passé correspondent-ils à ceux des locuteurs natifs ?

\section{Le développement des conjugaisons du passé}

Nous présenterons d'abord le développement progressif des différentes conjugaisons du passé dans la production des trois groupes d'apprenants du corpus. Ensuite nous comparerons l'emploi des conjugaisons du passé par le groupe d'apprenant le plus avancé (NN6) avec celui des natifs du même âge (FF6).

\subsection{Développement de la fréquence des conjugaisons du passé chez les apprenants FL2}

Les données relatives aux conjugaisons du passé, reprises dans le tableau 2, incluent l'emploi du passé composé, de l'imparfait et du plus-que-parfait. 
Tableau 2. Développement des conjugaisons du passé3

\begin{tabular}{|c|c|c|c|c|c|c|c|c|c|}
\hline & \multicolumn{3}{|c|}{ NN1 } & \multicolumn{3}{|c|}{ NN3 } & \multicolumn{3}{|c|}{ NN6 } \\
\hline & $\#$ & \%Fpassé & $\%^{\text {totV }}$ & \# & \%Fassé & $\%^{\text {totV }}$ & $\#$ & \%Fpassé & $\%^{\text {totV }}$ \\
\hline passé composé & 7 & 1,00 & 0,02 & 35 & 0,80 & 0,06 & 86 & 0,72 & 0,07 \\
\hline imparfait & 1 & & 1 & 9 & 0,20 & 0,01 & 23 & 0,19 & 0,02 \\
\hline plus-que-parfait & 1 & & / & 1 & 1 & 1 & 11 & 0,09 & 0,01 \\
\hline Total passé & 7 & 1,00 & $\mathbf{0 , 0 2}$ & 44 & 1,00 & $\mathbf{0 , 0 7}$ & 120 & 1,00 & $\mathbf{0 , 1 0}$ \\
\hline
\end{tabular}

Les trois conjugaisons du passé se développent progressivement, passant de $2 \%$ des formes verbales produites à $10 \%$ dans le groupe NN6 (le test non-paramétrique KruskalWallis indique que ce développement de la fréquence relative $\%$ totV est statistiquement significatif, étant donné que la valeur $\mathrm{p}$ est inférieure au seuil de $0.05, \mathrm{p}=0.000$ ). Dans le corpus NN1, elles se concrétisent uniquement sous la forme du passé composé. Les sept occurrences représentent $2 \%$ du nombre total de formes verbales produites. L'imparfait apparaît timidement dans le corpus $\mathrm{NN} 3$, apparition qui va de pair avec un emploi plus productif du passé composé $\left(6 \%{ }^{\mathrm{totV}}\right)$. Ce développement du passé composé est statistiquement significatif $(\mathrm{p}=0.002)$. L'emploi de la morphologie du passé est plus varié et plus riche dans le corpus NN6, d'une part par l'emploi plus productif de l'imparfait, qui double sa fréquence par rapport au nombre total de formes verbales produites, mais également par l'apparition d'occurrences au plus-que-parfait. Le développement du plusque-parfait entre les groupes NN3 et NN6 est statistiquement significatif $(p=0.026)$, mais il ne l'est pas pour l'imparfait $(\mathrm{p}=1.000))$.

Tableau 3. Répartition des occurrences du passé composé et de l'imparfait

\begin{tabular}{|l|c|c|c|c|c|c|}
\cline { 2 - 7 } \multicolumn{1}{c|}{} & \multicolumn{3}{c|}{ Passé composé } & \multicolumn{3}{c|}{ Imparfait } \\
\cline { 2 - 8 } \multicolumn{1}{c|}{} & NN1 & NN3 & NN6 & NN1 & NN3 & NN6 \\
\hline \# formes & 7 & 35 & 86 & 0 & 9 & 23 \\
\hline \% apprenants produisant formes & $17 \%$ & $68 \%$ & $94 \%$ & $/$ & $20 \%$ & $31 \%$ \\
\hline \# moyen/participant & 0,2 & 1,4 & 2,7 & $/$ & 0,4 & 0,7 \\
\hline Écart-type & 0.63 & 1.5 & 2.13 & $/$ & 0.86 & 1.55 \\
\hline \# maximal formes/ participant & 3 & 6 & 8 & $/$ & 3 & 7 \\
\hline
\end{tabular}

Le pourcentage d'apprenants produisant des formes conjuguées au passé composé et à l'imparfait augmente, ainsi que le nombre de formes produites par narration. La tendance est beaucoup plus nette pour le passé composé que pour l'imparfait, ce qui s'explique par le fait que le processus s'amorce bien plus tôt dans le premier cas. Ainsi, alors qu'une minorité d'apprenants utilise l'imparfait au sein du groupe le plus avancé, la quasi-totalité de ceux-ci produit des occurrences du passé composé.

Les valeurs de l'écart-type sont très basses et relativement stables, augmentant très légèrement pour l'emploi du passé composé et indiquant donc que ces données sont un peu plus dispersées dans le corpus NN6 que dans le corpus NN3. Quatre des cinq apprenants NN1 et presque la moitié des apprenants du groupe NN3 (47\%) qui produisent des occurrences du passé composé n'en produisent qu'une seule, mais dès NN6, 60\% des apprenants en

\footnotetext{
${ }^{3}$ Le taux de corrélation pour les variables $\%$ totmots (fréquence relative par rapport au nombre total de mots produits) et $\%$ totV (fréquence relative par rapport au nombre total de verbes produits) est tellement élevé que seuls les résultats pour \% ${ }^{\text {totV }}$ seront présentés dans les tableaux suivants.
} 
produisent au moins deux. Par contre $60 \%$ des utilisateurs de l'imparfait en NN6 ne l'emploient toujours qu'une seule fois. Bref, l'extension progressive de l'emploi des conjugaisons du passé est lente mais réelle au sein des groupes d'apprenants FL2 et cela tend à augmenter légèrement la différenciation entre apprenants, ce qui transparaît particulièrement au sein du groupe NN6.

Dans NN6, La grande majorité des verbes conjugués au passé composé sont des verbes à thème unique, réguliers, tels trouver ou chercher $(53 \%$, ex. 1) et des verbes à plusieurs thèmes sans variation vocalique tels partir ou dire (31\%, ex. 2) (cf. Michot 2014 pour la classification morphologique des verbes ${ }^{4}$ ).

(3) a. NN6-621: derrière un arbre qui est tombé sur la terre

b. NN6-630: le garçon a trouvé un petit animaux

(4) a. NN6-620: il découvre que le grenouille est parti

b. NN6-633: il a dit contre le chien de taire parce que il entend quelque chose

Quant aux conjugaisons à l'imparfait, les verbes sans tronc (avoir, être et aller) (55\%, ex. $6)$ et les verbes à thème unique $(32 \%$, ex. 7$)$ sont les sous-classes verbales les plus productives.

(6) a. NN6-Le13 : il pense que la grenouille était là

b. NN6-Sn13: le garçon allait au lit

(7) a. NN6-Me20: ils cherchaient partout dans la chambre

b. NN6-Sn10 : il l'aimait beaucoup

Les apprenants du groupe NN6 produisent pour la première fois des formes verbales conjuguées au plus-que-parfait. Il s'agit d'onze occurrences, essentiellement de verbes à thème unique $(64 \%$, ex. 8$)$ ou de verbes à plusieurs thèmes sans variation vocalique $(27 \%$, ex. 9). Une seule occurrence provient d'un verbe à plusieurs thèmes avec variation vocalique (faire) (ex. 10).

(8) a. NN6-812: ils voient que c'est le grenouille qu'ils avaient attrapé l'autre jour b. NN6-Me20 : ils avaient trouvé une grenouille

(9) a. NN6-633 : à un moment le petit garçon voit que le grenouille était parti

b. NN6-Sn7: le grenouille avait disparu

(10) NN6-Sn7 : le chien avait fait tomber ce ruche d'abeilles

\subsection{Fréquence des conjugaisons du passé chez les apprenants NN6 et les natifs FF6}

Nous examinons la fréquence d'emploi des conjugaisons du passé par le groupe d'apprenants le plus avancé (NN6) avec celui des natifs du même âge (FF6).

\footnotetext{
${ }^{4}$ La catégorisation proposée dans Michot 2014, basée sur la conjugaison à l'oral et inspirée Pouradier Duteil (1997), comprend quatre types de verbes : (a) les verbes à thème unique, (b) les verbes à plusieurs thèmes sans variation vocalique, (c) les verbes à plusieurs thèmes avec variation vocalique et (d) les verbes sans tronc. Les verbes à thème unique n'ont qu'un seul thème auquel s'attachent les différents suffixes. Ils correspondent aux verbes classés traditionnellement parmi les verbes de la première conjugaison. Les verbes des catégories (b) et (c) ont plusieurs thèmes, dont des thèmes qui se distinguent les uns des autres sans qu'une variation vocalique ne s'opère (ex. [par] et [part] du verbe partir) et ceux dont les différents thèmes se distinguent par une variation vocalique (ex. [pØ], [puv] et [pœv] du verbe pouvoir). Les verbes sans tronc sont les verbes être, avoir et aller dont les différentes formes verbales n'ont pas un seul phonème en commun.
} 
Tableau 4. Comparaison de la fréquence d'emploi des conjugaisons du passé chez les apprenants NN6 et natifs FF6

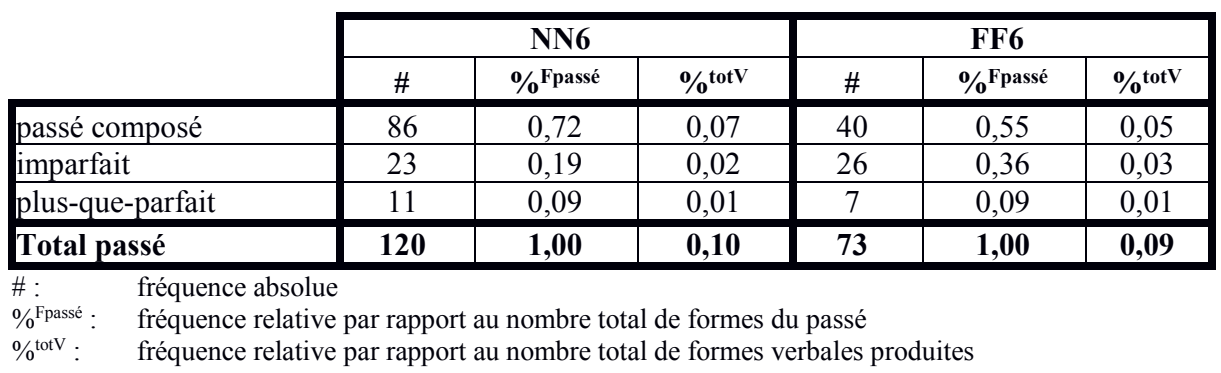

Rappelons d'abord que, si la longueur de la production est comparable entre le groupe le plus avancé d'apprenants (NN6) et les natifs du même âge (moyenne de 329 mots (NN6) par rapport à 369,2 mots (FF6) par participant), un écart important apparaît dans la fréquence d'emploi de formes verbales (40 verbes par apprenant NN6 contre 59 verbes par locuteur natif FF6) (cf. tableau 1). Cependant, le pourcentage d'emploi global des conjugaisons du passé par rapport au nombre total de verbes produits est similaire dans FF6 et dans NN6 (9\% vs 10\%). Sur le plan de la variété des conjugaisons verbales employées, la différence apprenants/ natifs est très limitée car tant les apprenants NN6 et les natifs FF6 produisent des occurrences conjuguées au passé composé, à l'imparfait et au plus-que-parfait.

Une différence saillante entre apprenants et natifs est la fréquence d'utilisation de l'imparfait : cette conjugaison représente en effet 36\% des formes du passé chez les natifs pour seulement $19 \%$ chez les apprenants les plus avancés de notre corpus. La généralisation de l'emploi de l'imparfait parmi l'ensemble des locuteurs des groupes considérés distingue également les apprenants des natifs : 31\% des apprenants NN6 utilisent l'imparfait alors que $53 \%$ des natifs FF6 l'emploient dans leur production.

\subsection{Commentaires}

Que nous apprennent les données de l'étude à propos du degré d'affinement du rapport forme-fonction dans les productions des apprenants et du degré de convergence du rapport forme-fonction entre la production des apprenants et celle des natifs? Nous considérons successivement les deux plans :

1) L'évolution de la fréquence d'emploi des conjugaisons du passé dans la production des apprenants FL2.

L'examen du développement des conjugaisons du passé dans la production des trois groupes d'apprenants montre une progression systématique des conjugaisons du passé à partir du groupe le moins avancé vers le groupe le plus avancé, ainsi qu'une extension de leur emploi sur l'ensemble des apprenants du groupe. Cependant, dans le groupe le plus avancé, le passé composé est toujours la seule conjugaison largement utilisée par la grande majorité des apprenants.

2) La comparaison de la fréquence d'emploi des apprenants avancés et des natifs.

A première vue, l'emploi global des conjugaisons du passé est similaire entre les apprenants plus avancés et les natifs du même âge : le pourcentage d'emploi global des conjugaisons du passé par rapport au nombre total de verbes produits est similaire $(9 \%$ dans FF6 vs 10\% dans NN6). La seule différence notable est la fréquence d'utilisation de l'imparfait : cette conjugaison représente en effet $36 \%$ des formes du passé chez les natifs pour seulement $19 \%$ chez les apprenants les plus avancés de notre corpus. 
Cependant, une comparaison des productions des deux groupes de natifs FF1 et FF6 met en évidence que l'emploi des conjugaisons du passé par les natifs, lui aussi, ne reste pas constant mais varie assez considérablement.

Tableau 5. Développement des conjugaisons du passé chez les natifs

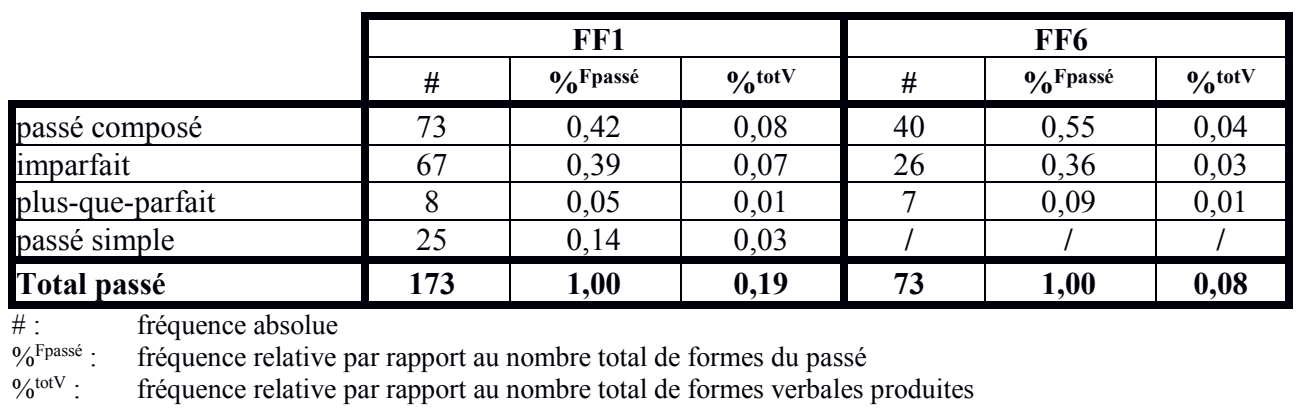

Ainsi, des différences remarquables apparaissent dans la comparaison de la production des deux groupes de locuteurs natifs : la fréquence d'emploi de l'ensemble des conjugaisons du passé est sensiblement inférieur dans les productions des natifs plus âgés ( $9 \%$ dans FF6 et $19 \%$ dans FF1). Une seconde différence remarquable est l'utilisation relativement fréquente du passé simple dans les productions des natifs FF1, qui n'apparaît pas dans les productions des natifs FF6. Les occurrences conjuguées au passé simple représentent $14 \%$ de la totalité des conjugaisons du passé produites ( $\left.\%{ }^{\text {Fpassé}}\right)$, 3\% de la totalité de verbes produits $\left(\%^{\text {totV }}\right)$. Cependant, la fréquence d'emploi totale des conjugaisons au passé composé et au passé simple dans FF1 correspond à la fréquence d'emploi du passé composé seul dans FF6 $( \pm 55 \%$ des formes du passé dans les deux corpus).

Les raisons de cette variation sont sans doute diverses et devront être examinées plus systématiquement dans divers contextes et pour diverses tâches. Elle pourrait par exemple être liée:

- à une motivation plus forte des jeunes locuteurs FF1 pour réaliser la tâche de narration dans un contexte scolaire et pour utiliser explicitement des conjugaisons enseignées pour réaliser une narration au passé (emploi du passé simple) ;

- à l'exploitation d'autres conjugaisons et donc à la convocation d'autres types de configurations narratives pour raconter l'histoire au passé.

Quoi qu'il en soit, les données révèlent que l'usage des conjugaisons du passé peut aussi varier chez les natifs sur la même tâche selon l'âge. Il ne semble donc pas exister de modèle de référence absolu auquel la fréquence d'emploi des apprenants L2 peut être comparée. Ces constatations pertinentes invitent (a) à porter un nouveau regard sur le développement des conjugaisons du passé chez les apprenants L2, qui ne doit pas nécessairement être linéaire et appréhendé uniquement du point de vue de la fréquence, (b) à réexaminer la fréquence d'emploi de ces conjugaisons par les natifs, qui n'est pas invariable, mais qui requiert la prise en considération de nouveaux paramètres tels l'âge, le scénario narratif, l'exploitation de différentes combinaisons de conjugaisons, etc. Plus particulièrement, le recul sensible de l'exploitation de conjugaisons du passé chez les natifs du groupe FF6 met en évidence que l'expression du passé ne passe pas nécessairement par l'utilisation des conjugaisons du passé et que leur maîtrise n'équivaut pas à un emploi systématique de celles-ci dans tous les contextes passés. Elle peut aussi être liée à l'exploitation d'autres conjugaisons et donc à la convocation d'autres types de configurations narratives pour raconter l'histoire au passé, configurations que nous examinons en détail dans le prochain volet de l'étude. 


\section{Les configurations narratives pour l'exploitation des conjugaisons du passé}

Le développement de la maîtrise des conjugaisons du passé mais aussi la disponibilité d'autres formes pour les mêmes types de fonctions multiplie les combinaisons de conjugaisons possibles dans divers types de scénarios pour narrer la même histoire. Cette section décrit les diverses combinaisons de conjugaisons utilisées par les apprenants FL2 pour développer leur narration au passé et les compare avec celles utilisées par les natifs.

\subsection{Analyse des données FL2}

L'étude des productions des apprenants du groupe le plus avancé (NN6) révèle l'activation de plusieurs combinaisons temporelles possibles pour l'expression d'une narration au passé.

$1^{\circ}$ narrations centrées autour du présent

Dans la plupart des productions des apprenants du groupe NN6, la narration reste centrée autour de l'emploi du présent, avec éventuellement l'apport additionnel d'une des conjugaisons du passé.

\section{a. narration sans conjugaisons du passé}

Dans plusieurs narrations, les conjugaisons du passé sont totalement absentes et l'histoire est racontée au présent. Parfois, le contexte ne permet pas de décider si cela découle d'une difficulté à positionner l'histoire dans le passé, mais dans d'autre cas, comme dans l'exemple (11), la configuration est clairement utilisée pour situer l'histoire au passé :

(11) Me-19: dans la nuit quand le garçon et le chien $\operatorname{dort}\left[{ }^{*}\right]$, la grenouille part du bocal

b. présent + passé composé

Cette deuxième configuration narrative représente la combinaison de conjugaisons la plus fréquente dans la production du groupe NN6. Dans ce type de configuration, le passé composé tend généralement à marquer le perfectif :

(12) a. NN6-626: il voit que la grenouille est partie

b. NN6-621: le jour après et la grenouille a disparu

le petit garçon s'éveille et il voit que la grenouille est s'enfui [*]

\section{c. Présent + imparfait}

En exploitant tout en élargissant l'hypothèse discursive de Bardovi-Harlig ( « learners use emerging verbal morphology to distinguish foreground from background in narratives » (1994 : 43)) par la prise en considération du présent, il est possible de relever une série d'énoncés qui expriment une opposition discursive plus généralisée, où l'arrière-plan est identifié par l'imparfait et le premier plan par le présent. Le corpus NN6 contient un certain nombre de configurations de ce genre (ex. 13) :

(13) a. NN6-815: quand il dormait le grenouille s'échappe dans le vitraux dans la fenêtre

b. NN6-Le13 : il pense que la grenouille était là alors il voit une animaux qui n'est pas son grenouille

c. NN6-Le17: le chien $v a$ avec son tête dans le pot où le grenouille était chaque soir mais il ne peut échapper de ce pot

d. NN6-Sn7 : quand il allait dormir sa grenouille s'enfuit

e. NN6-Sn7 : $\quad$ les abeilles fuient le chien parce qu'il était très embêtant

f. NN6-Sn13 : le soleil commençait à briller et le petit garçon Jean se réveille 
$2^{\circ}$ Narrations basée sur la combinaison de temps du passé

$\mathrm{Ce}$ cas de figure est extrêmement rare dans la production des apprenants du groupe d'apprenants le plus avancé. Cependant, deux apprenants NN6 produisent une séquence de conjugaison combinant l'imparfait et le plus-que-parfait, même si elles contiennent aussi des formes conjuguées au présent et au passé composé :

(14) a. NN6-Me20 : il y avait un petit garçon et un chien et ils avaient trouvé une grenouille

\section{ils l'avaient mis dans un boîte mais le lendemain il était disparu \\ alors ils cherchaient partout dans la chambre, mais ils ne le trouvaient pas \\ alors ils décidaient d'aller le chercher et ils crient pour la grenouille, mais ils ne la trouvent pas}

b. NN6-Sn13 : il y avait une fois un garçon et son petit chien Pluto qui avait attrapé une grenouille

$a$

il l'appelait Fien

il était assez tard tard et le garçon allait au lit et pendant la nuit Fien

su échapper par la

fenêtre

le soleil commençait à briller et le petit garçon se réveille

\section{$3^{\circ}$ Narrations combinant le présent avec diverses conjugaisons du passé}

Ce type de configuration est nettement plus complexe et permet d'exprimer divers types de nuances dans le cadre d'une narration au passé. Il s'agit plus spécifiquement de la combinaison présent + passé composé + imparfait. Une telle exploitation de trois conjugaisons au sein d'une même configuration temporelle est rare dans la production NN6 :

(15) a. NN6-628: et la nuit le grenouille il échappe [*] et le lendemain le petit gamin il $a$ vu que le grenouille mais il ne le voit plus

n'était pas là

il a regardé dans tous ses vêtements et le bocal et ensuite son lit

b. NN6-Me20: et il tombe sur une grand pierre et il fait Fifi Fifi

en criant Fifi il tenait des cornes d'un cerf

tête

et alors le cerf élevait la tête et le petit garçon est tombé sur son

c. NN6-Sn13: il était assez tard et le garçon allait au lit et pendant la nuit Fien $a$ su échapper par la fenêtre

Dans ce cas de figure, à l'instar du contraste entre le présent et le passé composé, l'opposition passé composé / imparfait peut marquer une opposition aspectuelle (imperfectif/ perfectif). Parfois aussi, l'opposition des deux conjugaisons tend à mettre en évidence une opposition discursive en distinguant le premier plan et l'arrière-plan (cf. Bardovi-Harlig 1994). Ce dernier emploi apparaît dans le corpus NN6, mais reste limitée à quelques rares contextes :

(16) a. NN6-814 : *PUP : et sa tête est restée dans le bocal, il était

*INV : bloqué

*PUP : bloqué

b. NN6-814 : $\quad$ il a sauté du fenêtre et tombE [*] et comme ça le bocal était cassé

L'analyse détaillée des productions des apprenants NN6 permet de conclure que la production de la narration au passé est réalisée au moyen de diverses configurations narratives. Si la variété des combinaisons de conjugaisons possibles est remarquable et 
commence à présenter un certain niveau de sophistication, le présent joue néanmoins souvent un rôle central au sein de celles-ci.

\subsection{Analyse des données FL1}

Une large variété de configurations narratives apparaît également dans les productions des natifs.

\section{$1^{\circ}$ narrations centrées autour du présent}

La narration centrée autour de l'emploi du présent, avec éventuellement l'apport additionnel d'une des conjugaisons du passé, est également fréquente dans la production des natifs.

\section{a. narration sans conjugaisons du passé}

Chez les natifs également, diverses narrations sont produites exclusivement au présent. La dimension temporelle dans ces narrations est néanmoins souvent assurée par d'autres marques dans le discours, telles des connecteurs ou des circonstants (les éléments localisant clairement la narration au passé sont soulignés dans l'exemple 17).

a. FF1-727:

alors $c$ 'est un petit garçon qui regarde une grenouille avec le

chien

pendant la nuit la grenouille elle s'en va

le matin le garçon il se réveille

il voit plus la grenouille

alors il cherche

il s(e) habille

il cherche

le petit chien il met sa tête dans le bocal

il est coincé

le petit garçon il appelle la grenouille

le petit chien il tombe

après le petit garçon il ramasse son chien

b. FF6-701 : il y a un petit garçon qui $a$ une grenouille apparemment et un chien et pendant la nuit la grenouille $s$ 'échappe

et il se réveille

il n'a plus de grenouille

donc il est atterré

donc il fouille un peu partout dans sa chambre apparemment

le chien il trouve une chose à faire

c'est mettre sa tête dans le bocal

puis le chien passe par la fenêtre

donc après ils partent

b. présent + passé composé

Cette configuration, qui est très fréquente chez les natifs tout comme chez les apprenants, semble indiquer une opposition aspectuelle, où le passé composé exprime le perfectif.

(18) a. FF1-716: alors c'est un petit garçon et il a attrapé une grenouille dans un bocal et le chien est émerveillé aussi et alors quand ils vont dormir il y a la grenouille qui sort de son bocal

b. FF6-688: il se couche 


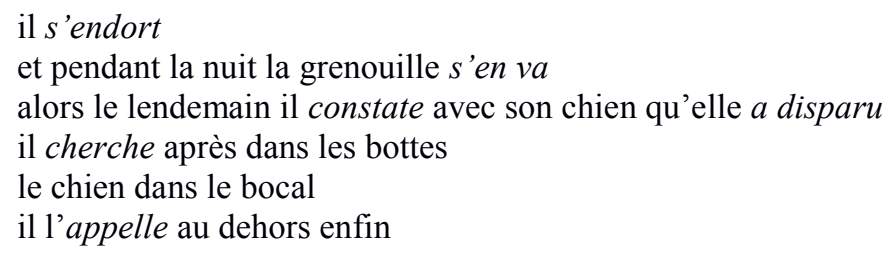

c. présent + imparfait

Cette troisième configuration est plus rare. Il s'agit d'une combinaison de conjugaisons où l'imparfait indique une action non conclue, engagée antérieurement et qui persiste au moment de l'énonciation (aspect imperfectif) :

(19) a. FF6-697: il essaie un peu de jouer avec, de l'attraper

le petit garçon qui cherchait dans un trou

se fait mordre par la bestiole qui se trouvait dedans

b. FF1-715 puis le petit garçon dit qu'il faut se taire au chien

et puis il cherche et puis il tombe sur des crapauds sur une famille de crapauds

le chien et le petit garçon étaient tout contents

parce qu'ils avaient trouvé les crapauds.

\section{$2^{\circ}$ Narrations basée sur la combinaison de temps du passé}

L'emploi systématique des deux conjugaisons du passé n'apparaît que chez les natifs. Différentes configurations peuvent être observées :

\section{a. passé composé + imparfait}

Cette combinaison sert souvent à marquer une opposition discursive située dans le passé, permettant au locuteur de distinguer le premier plan de l'arrière-plan :

(20) a. FF1-718 : Pendant ce temps là le chien était en train d'encore jouer et il a fait tombé la ruche

b. FF1-726: il était une fois un petit garçon qui aimait sa petite grenouille

il était dans sa chambre

et il faisait noir

et il devait aller dormir

pendant son sommeil la grenouille s'est enfuie

puis au matin il s'est réveillé

et il $a v u$ que sa grenouille $a$ disparu

il regardait un peu partout

\section{b. passé simple + imparfait}

L'utilisation du passé simple dans une configuration temporelle narrative est propre à la production des natifs (à celle du groupe FF1 dans notre cas). Elle marque prototypiquement l'opposition aspectuelle dans une époque passée : le passé simple marque ainsi une action accomplie (perfectif), tandis que l'imparfait exprime une action en accomplissement (imperfectif). L'époque passée est soulignée dès le début de la narration par les circonstants un jour et il était une fois :

(21) a. FF1-720: un jour un petit garçon et son chien trouvèrent une jolie petite grenouille qu'ils mirent précieusement dans un beau bocal le soir avant d'aller se coucher ils la contemplèrent 
pas là

et le lendemain au réveil du petit garçon et de son chien elle était

alors ils la cherchèrent partout dans les bottes

et ils crièrent après elle à la fenêtre

ils partirent en forêt

le petit garçon il inspectait tous les pas qu'il y avait par terre

b. FF1-728: $\quad$ il était une fois un petit garçon et un petit chien qui allaient se coucher très tard

un bocal.

parce qu'ils avaient une grenouille qu'ils avaient enfermée dans

ils la regardaient tous les soirs

un jour ils $s$ 'endormèrent

et la grenouille sort du bocal [//] ou sortit

un matin ils se réveillèrent

et la grenouille fut partie du bocal

ils cherchèrent partout

mais ils la trouvèrent pas

c. imparfait + plus-que-parfait

La combinaison convoquée souligne l'antériorité dans le passé d'un événement par rapport à un autre.

(22) FF1-717: c'est l'histoire d'un petit garçon et d'un petit chien qui contemplaient une grenouille qu'ils avaient peut-être capturée dans le lac

$3^{\circ}$ Narrations combinant le présent avec diverses conjugaisons du passé

Ce type de configuration, plus complexe, favorise l'expression de divers types de nuances dans le cadre d'une narration au passé. Il s'agit plus spécifiquement de la combinaison présent + passé composé + imparfait. Elle permet de combiner les distinctions aspectuelles (perfectif/ imperfectif) et discursives (avant/ arrière-plan) :

(23) a. FF1-722: et l'oiseau le frappe, si je peux dire

puis l'oiseau est parti

il remonte au dessus

puis il y avait je pensais un cerf qui dépassait ses cornes

le garçon $s$ 'est avancé

il est tombé sur ses cornes

le cerf est parti

enfin il laisse tomber par terre avec le chien dans l'eau

Dans l'exemple (23a), le locuteur exploite en effet l'opposition présent/ passé composé pour marquer l'opposition aspectuelle perfectif/ imperfectif et celle entre l'imparfait et les autres conjugaisons pour distinguer l'avant de l'arrière-plan. Une telle exploitation de trois conjugaisons au sein d'une même configuration temporelle est fort fréquente dans la production des natifs (cf. aussi 714FF1, 723FF1, 723FF1, 697FF6, 689FF6, 747FF6):

(23) b. FF1-723 : et le chien il courait [//] il court parce qu'il est poursuivi par le nid d'abeilles qu'il a ennuyé

alors cela a fait du bruit

une chouette est apparue dans l'arbre

et la petite est tombée

après la chouette $s$ 'est envolée

la petite $a$ eu peur.

puis elle est remontée sur un mont 
clairière

c. FF6-689 :

après le chien et elle a regardé de loin

et sur le mont elle croyait qu'il y avait des branches

mais c'étaient les cornes d'un cerf

elle $a$ été emportée

et le cerf il court

le cerf l'a fait tomber elle et son petit chien dans un genre de

puis ils sont tombés dans un marais

apparemment $c$ 'est une souris qui était dans le trou

donc il se tourne pour chercher autre part

le chien en attendant il a fait tomber le nid

et à ce moment-là y $a$ toutes les abeilles qui commencent à courir

le garçon lui il regardait dans un tronc parce qu'il avait $v u$ un trou

il voulait voir ce qui en était dedans

en fait $c$ 'était un hibou

et le hibou lui a fait peur

et il est tombé de l'arbre

et alors le hibou il va apparemment [//] il le pourchasse

et lui il va [//] il se met [//] il monte sur un caillou [*]

\subsection{Commentaires}

Plusieurs conclusions découlent des faits observés chez les apprenants et les natifs et de la comparaison de ceux-ci :

$1^{\circ}$ De l'aperçu descriptif de la manière de procéder des natifs, il ressort clairement qu'il n'y a pas une seule "configuration temporelle» pour raconter l'histoire de la grenouille. Par ailleurs, cette narration peut se faire sans avoir nécessairement recours à une des conjugaisons du passé.

$2^{\circ}$ Les apprenants les plus avancés de notre étude réalisent déjà la narration au passé au moyen de diverses configurations narratives. Si la variété des combinaisons de conjugaisons possibles est remarquable et commence à présenter un certain niveau de sophistication, le présent joue néanmoins souvent un rôle central au sein de celles-ci.

$3^{\circ} \mathrm{Ce}$ qui distingue sur le point des combinaisons de conjugaisons les natifs des apprenants NN6, c'est cependant la variété et la richesse des configurations narratives convoquées. Si certains de ceux-ci émergent déjà dans la production des apprenants NN6, c'est encore sous une forme embryonnaire et hésitante.

Une étude plus systématique et plus large des configurations narratives devrait permettre de les lier plus clairement aux différents types de points de vue adoptés par le locuteur pour réaliser la tâche.

\section{Conclusions}

Les données examinées et analysées dans l'étude permettent de dégager quelques tendances qui caractérisent le développement de l'appariement forme-fonction en FL2.

Notre contribution montre que la fréquence d'emploi des conjugaisons du passé progresse systématiquement dans la production des divers groupes d'apprenants FL2 et que leur emploi s'étend sur l'ensemble des apprenants du groupe, même si le passé composé est la seule conjugaison largement utilisée par la grande majorité des apprenants du groupe avancé. De plus, le pourcentage d'emploi global des conjugaisons du passé par rapport au nombre total de verbes produits est similaire dans FF6 et dans NN6. Sur le plan de la variété des conjugaisons verbales employées, la différence apprenants/ natifs est en fin de compte 
limitée, sauf pour la fréquence d'utilisation de l'imparfait, nettement plus basse chez les apprenants (et l'emploi du passé simple, propre aux natifs).

Le degré d'affinement du rapport forme-fonction dans la production des apprenants progresse donc systématiquement et le degré de convergence du rapport forme-fonction entre apprenants et natifs s'accroît, même si des différences pertinentes subsistent, en particulier pour certaines conjugaisons du passé

L'examen des types de configurations narratives exploités par le groupe d'apprenants le plus avancé dans notre étude révèle qu'ils réalisent déjà la narration au passé au moyen de combinaisons diverses de conjugaisons. La diversification de celles-ci commence à présenter un certain niveau de sophistication, même si le présent joue souvent un rôle central en leur sein. Cependant, la variété et la richesse des configurations convoquées pour réaliser la narration au passé distinguent encore la production des natifs de celle des apprenants.

Dans la manière dont diverses conjugaisons sont convoquées pour l'expression d'une fonction spécifique, l'expression d'une narration au passé, les données signalent l'importance de prendre en considération la multiplicité des combinaisons de conjugaisons et de leur exploitation dans divers types de configurations narratives pour raconter la même histoire. De l'analyse de manière de procéder des natifs, il ressort en effet qu'il n'y a pas un seul « scénario » imposé pour raconter l'histoire de la grenouille. Par ailleurs, cette narration peut se faire sans avoir nécessairement recours à une des conjugaisons du passé.

En conclusion, les résultats de l'étude mettent en garde contre une vision unilatérale de la progression de la maîtrise en L2 concernant l'expression du passé, qui serait associée à un emploi de plus en plus généralisé et fonctionnellement approprié des conjugaisons du passé. La perspective temporelle dans une narration peut être développée à partir de différentes combinaisons de conjugaisons et de différents points de vue narratifs. Dans ce contexte, notre contribution souligne aussi l'importance de référer systématiquement aux usages de groupes de natifs pour chaque type de production et de tâche langagière spécifique.

\section{Références}

Andersen, R. W. (1984). The one-to-one principle of interlanguage construction. Language Learning, 34, 77-95.

Andersen, R. W. (1986). El desarrollo de la morfologia verbal en el español como segundo idioma. In Meisel, J. (Ed.), Adquisición de lenguaje/Aquisição da linguagem, (pp. 115-138). Frankfurt : Vervuert.

Andersen, R. W. (1990). Models processes, principles and strategies: Second language acquisition inside and outside the classroom. In Bill VanPatten \& James F. Lee (eds.), Second language acquisition-foreign language learning, (pp. 45-78). Clevedon, UK: Multilingual Matters.

Andersen, R. W. (1991). Developmental sequences : the emergence of aspect marking in second language acquisition. In Huebner, T., \& Ferguson, C. (eds.), Crosscurrents in second language acquisition and linguistic theories. Amsterdam : John Benjamins. 305-324.

Ayoun, D. (2001). The role of negative and positive feedback in the second language acquisition of passé composé and imparfait. Modern Language Journal, 85, 226-243. 
Ayoun, D. (2004). The Effectiveness of Written Recasts in the Second Language Acquisition of Aspectual Distinctions in French : A Follow-Up Study. The Modern Language Journal, $88,31-55$.

Bardovi-Harlig, K. (1994). Anecdote or evidence ? Evaluating support for hypotheses concerning the development of tense and aspect. In Tarone, E., Gass, S. M., Cohen, A. D., Research methodology in second language acquisition. Hillsdale : Erlbaum. 243-282.

Comrie, B. (1976). Aspect. An introduction to the study of verbal aspect and related problems. Cambridge : Cambridge University Press.

Granget, C. (2005). Développement de l'accord verbal avec un sujet pluriel dans les récits écrits d'apprenants germanophones scolarisés du français. In : Granfeldt, J. \& Schlyter, S.(eds.), Acquisition et production la morphologie flexionnelle, Perles 20 (pp. 111-123). Université de Lund.

Kaplan, M. A. (1987). Developmental patterns of past tense acquisition among foreign language learners of French. In VanPatten, B., Dvorak, T. R., Lee, J.F. (eds.), Foreign language learning : a research perspective (pp. 52-60). Cambridge, MA : Newbury House.

Kihlstedt, M. (2002). L'emploi de l'imparfait en français par des locuteurs suédophones et des locuteurs natifs. Revue française de linguistique appliquée, VII (2), 7-23.

Klein, W. (1995). Frame of analysis. In Dietrich, R., Klein, W., Noyau, C.(eds.), The acqusition of temporality in a second language, (pp. 17-29). Amsterdam : John Benjamins.

Labeau, E. (2005). Des «Temps modernes »: L'aspect suffit-il à la maîtrise des temps du passé dans les narrations écrites d'apprenants avancés ?. In Molendijk, A. \& Vet, C., Temporalité et attitude: structuration du discours et expression de la modalité. Cahiers Chronos, 12, (pp. 203-218). Amsterdam : Rodopi.

Li, P. \& Shirai, Y. (2000). The Acquisition of Lexical and Grammatical Aspect. Berlin : Mouton De Gruyter.

MacWhinney, B. (2000). The CHILDES Project : Tools for Analyzing Talk. $3^{\text {rd }}$ edition. Mahwah, NJ : Lawrence Erlbaum.

Mayer, M. (1969). Frog, where are you? New-York : Dial.

McManus, K. (2015). L1-L2 Differences in the Acquisition of Form-Meaning Pairings in a Second Language. Canadian Modern Language Review, 71 (2), 155-181.

Michot, M.-E. (2014). L'acquisition de l'accord sujet-verbe en FLE : l'impact de la classe verbale. In Michot, M.-E.(Ed.), L'acquisition de la morphologie verbale en FLE, Cahiers de l'AFLS, 19 (1), 57-79. 
Michot, M.-E. \& Pierrard, M. (2016). L'appariement forme-fonction en FL2: le développement de l'emploi des formes verbales du passé et la primauté du marquage de

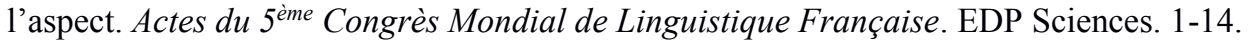

Noyau, C. (1997). Processus de grammaticalisation dans l'acquisition de langues étrangères : la morphologie temporelle. In Martinot, C. (Ed.), L'acquisition de la syntaxe, (pp. 223-252). Besançon : Annales littéraires de l'Université de Franche-Comté

Noyau, C., Houdaifa, E.-T., Vasseur, M.-T., Véronique, D. (1995). The acquisition of French. In Dietrich, R., Klein, W., Noyau, C. (eds.), The acquisition of temporality in second language, (pp. 145-209). Amsterdam : John Benjamins.

Perdue, C., Benazzo, S., Giuliano, P. (2002). When finiteness gets marked : the relation between morphosyntactic development and use of scopal items in adult language acquisition. Linguistics, 40 (4), 849-890.

Perdue, C. \& Klein, W. (1992). Why does the production of some learners not grammaticalize? Studies in Second Language Acquisition, 14, 259-272.

Salaberry, R. (1999). The development of past tense verbal morphology in classroom L2 Spanish. Applied Linguistics, 20, 151-178.

Salaberry, R. (2003). Tense Aspect in Verbal Morphology. Hispania, 86 (3), 559-5573.

Salaberry, R., \& Ayoun, D. (2005). The development of L2 tense-aspect in the Romance languages. In Ayoun, D., \& Salaberry, R., Tense and aspect in Romance languages. Amsterdam : John Benjamins. 1-33.

Thomas, A. (2009). Les débutants parlent-ils à l'infinitif? Influence de l'input sur la production des verbes par des apprenants adultes du français. Thèse de doctorat, Université de Lund.

Véronique, D. (2009). L'acquisition de la grammaire du français, langue étrangère. Paris : Didier. 